\title{
NEXT-GENERATION SEQUENCING IDENTIFIES A HOMOZYGOUS NONSENSE P.TYR370* MUTATION OF THE TMC6 GENE IN A MEXICAN PEDIGREE WITH EPIDERMODYSPLASIA VERRUCIFORMIS
}

\author{
Samantha López-RamíreZ ${ }^{1}$, Yuritzi Santillán-HernándeZ ${ }^{1}$, Eugenio Carrasco-Gerard², \\ Agustín RodAs-SERRANO ${ }^{3}$, AND JUAN C. ZeNTENO ${ }^{4,5 *}$ \\ ${ }^{1}$ Genetics Program, Faculty of Medicine, Universidad Nacional Autónoma de México (UNAM) and Centro Médico \\ Nacional "20 de Noviembre" (ISSSTE), Mexico City; ${ }^{2}$ Dermatology Program, Faculty of Medicine, UNAM and Hospital \\ General de México "Dr. Eduardo Liceaga", Mexico City; ${ }^{3}$ Genetics and Molecular Biology Service, Mount Sinai \\ Hospital, Cuenca, Ecuador; “ ${ }^{2}$ epartment of Genetics-Research Unit, Institute of Ophthalmology "Conde de Valenciana”, \\ Mexico City; ${ }^{5}$ Department of Biochemistry, Faculty of Medicine, UNAM, Mexico City, Mexico
}

\section{BACKGROUND}

Epidermodysplasia verruciformis (EV) is a rare genodermatosis characterized by abnormal susceptibility to infection with b-genotype human papillomavirus (HPV) and a particular propensity to develop cutaneous malignancies. Clinical manifestations include flat, scaly, reddish hypo- and hyperpigmented macules, verruca-like papillomatous lesions, seborrheic keratosis-like lesions, and pink-red pityriasis versicolor-like lesions ${ }^{1}$. EV patients develop non-melanoma skin cancers on areas of ultraviolet-exposed skin, mostly squamous-cell carcinomas, during the fourth or fifth decade of life. The increased carcinogenesis risk is probably caused by HPV infection, particularly highrisk HPV types 5 and $8^{2}$.

EV arises most commonly (60-75\% of cases) from recessive mutations in either $T M C 6$ or $T M C 8$, two closely related genes encoding integral membrane proteins that localize to the endoplasmic reticulum and are predicted to form transmembrane channels. TMC6 is expressed in keratinocytes, in which they control the intracellular levels of zinc ${ }^{3}$. In addition, at least other ten genes have been associated with the uncommon atypical EV form ${ }^{3}$. Here, we present the clinical and genetic features of two related EV patients in whom a causal TMC6 mutation was readily demonstrated by massive DNA sequencing.

\section{METHODS}

The proband (patient \#1) is a 44-year-old woman who was born in a small village in southern Mexico ( 2600 inhabitants). She presented with a generalized dermatosis affecting frontal, temporal and scalp regions, neck, shoulders, chest, back, arms, and thighs.
*Corresponding author:

Juan C. Zenteno

E-mail: jczenteno@institutodeoftalmologia.org
Received for publication: 18-08-2020

Approved for publication: 26-10-2020

DOI: $10.24875 / R I C .20000415$

0034-8376 / (c) 2020 Revista de Investigación Clínica. Published by Permanyer. This is an open access article under the CC BY-NC-ND license (http://creativecommons.org/licenses/by-nc-nd/4.0/). 
Figure \#1. Partial Sanger sequencing of exon 10 of the TMC6 gene. A homozygous C > G transversion at nucleotide 1110 was demonstrated in DNA from patient \#1 (A). This variant predicts a non-sense p.Tyr370* substitution at the EVER1 protein. Genetic analysis demonstrated heterozygosity for the mutation in the healthy father (carrier) of patient \#2 (B).
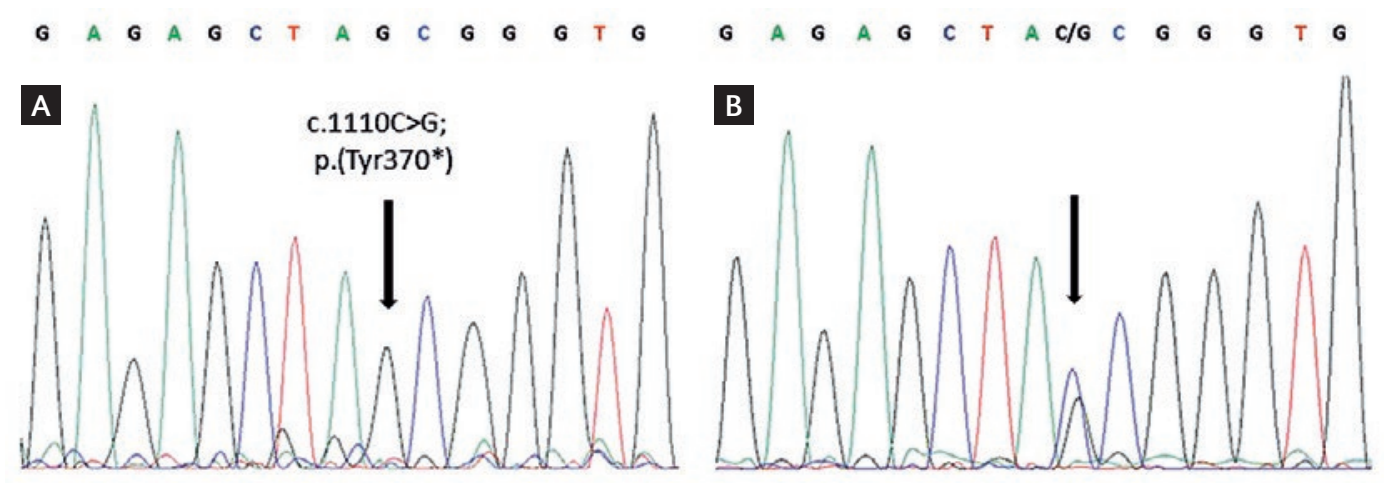

Skin lesions were warty, non-pruritic, hyperkeratotic, and mostly reddish-brown (Supplemental Fig. 1S, A-C). Skin lesions appeared at the age of 7 years. She had a history of parental consanguinity and at least other five living relatives were reported as affected but not available for examination. She developed skin neoplasias (squamous and basal cell carcinoma) which were treated with surgery and radiotherapy. The patient \#2 (Supplemental Fig. D-F), a cousin of patient \#1, is a 45 -year-old male who began his current condition at 7 years of age, presenting flat wart skin lesions distributed throughout the body, predominantly in areas with sun exposure, as well as macules and achromic plaques, mainly on the back and extremities. He began his assessment at the age of 11 years when he underwent his first resection of squamous cell carcinoma.

Given the association of EV to at least 13 genes, the genetic diagnosis was performed using next-generation sequencing (NGS) approach. Genomic DNA was isolated from blood leukocytes using standard procedures. NGS of 4813 genes associated with Mendelian genetic diseases was performed in proband's DNA using the Trusight One system (Illumina Inc., San Diego, CA, USA), employing the Miseq platform (Illumina Inc.). The downstream variant analysis was done with VariantStudio software (Illumina).

\section{RESULTS}

After NGS data analysis, a homozygous c. $110 \mathrm{C}>\mathrm{G}$ transversion at exon 10 of the TMC6 gene was

identified in DNA of the patient \#1 (Fig. \#1A). This variant is predicted to result in a non-sense p.Tyr370* change at the protein level. The variant was confirmed by Sanger sequencing in DNA from patients \#1 and \#2. This variant was classified as pathogenic according to the ACMG criteria. As expected, Sanger sequencing revealed that parents of the patient \#2 were heterozygous carriers for the p.Tyr370* mutation (Fig. \#1B). No DNA samples from other relatives were available for genetic analysis.

\section{DISCUSSION}

To the best of our knowledge, this is the first report of molecularly diagnosed EV patients from Mexico and Latin America. NGS readily identified a homozygous mutation, c.1110C > G; p.Tyr370*, in the TMC6 gene. Interestingly, an identical pathogenic variant in TMC6 was very recently demonstrated in an EV family of French ancestry ${ }^{3}$, which corresponded to family 3 in an early clinical report by Lutzner et al. ${ }^{4}$ As no history of European ancestors was recorded in the present family, it is possible that the p.Tyr370* mutation arose independently in these two pedigrees.

While approximately 500 EV cases have been described to date, it is likely that the disease is underdiagnosed due to inadequate clinical suspicion. In Mexico, there are few cases of patients reported with an $\mathrm{EV}$ diagnosis ${ }^{5}$ although no molecular diagnosis has been performed in any of them. While it has been stated that mutations in TMC6 and TMC8 account 
for as many as $60 \%$ of $E V$ cases $^{3}$, the true prevalence of EV-causing mutations is not known due to possible underreporting of mutation-negative cases and to the lack of large cohorts of molecularly analyzed patients. The potential identification of recurrent or founder mutations in a specific population is important because testing for one or a few prevalent mutations is more efficient and low-cost than testing for many rare variants.

\section{SUPPLEMENTARY DATA}

Supplementary data are available at Revista de Investigación Clínica online (www.clinicalandtranslationalinvestigation.com). These data are provided by the corresponding author and published online for the benefit of the reader. The contents of supplementary data are the sole responsibility of the authors.

\section{REFERENCES}

1. Huang S, Wu JH, Lewis DJ, Rady PL, Tyring SK. A novel approach to the classification of epidermodysplasia verruciformis. Int J Dermatol. 2018;57:1344-50.

2. de Jong SJ, Imahorn E, Itin P, Uitto J, Orth G, Jouanguy E, et al. Epidermodysplasia verruciformis: inborn errors of immunity to human beta-papillomaviruses. Front Microbiol. 2018;9:1222.

3. Youssefian $L$, Vahidnezhad $H$, Mahmoudi $H$, Saeidian $A H$, Daneshpazhooh $\mathrm{M}$, et al. Epidermodysplasia verruciformis: genetic heterogeneity and EVER1 and EVER2 mutations revealed by genome-wide analysis. J Invest Dermatol. 2019; 139:241-4.

4. Lutzner MA, Blanchet-Bardon C, Orth G. Clinical observations, virologic studies, and treatment trials in patients with epidermodysplasia verruciformis, a disease induced by specific human papillomaviruses. J Invest Dermatol. 1984;83:18-25

5. Ponce OR, Peniche RJ, Tirado SA, Arellano MI, Peniche CA, León DG, et al. Epidermodisplasia verruciforme. Modelo de carcinogénesis viral. Dermatol Rev Mex. 2006;50:83-6. 